\title{
Front propagation in hyperbolic fractional reaction-diffusion equations
}

\author{
Vicenç Méndez ${ }^{1}$ and Vicente Ortega-Cejas ${ }^{2}$ \\ ${ }^{1}$ Departament de Medicina, Facultat de Ciències de la Salut, Universitat Internacional de Catalunya, c/ Josep Trueta s/n, \\ E-08190 Sant Cugat del Vallès, Barcelona, Spain \\ ${ }^{2}$ Grup de Física Estadística, Departament de Física, Universitat Autònoma de Barcelona, E-08193 Bellaterra, Barcelona, Spain \\ (Received 2 April 2004; revised manuscript received 20 December 2004; published 31 May 2005)
}

\begin{abstract}
From the continuous-time random walk scheme and assuming a Lévy waiting time distribution typical of subdiffusive transport processes, we study a hyperbolic reaction-diffusion equation involving time fractional derivatives. The linear speed selection of wave fronts in this equation is analyzed. When the reaction-diffusion dimensionless number and the fractional index satisfy a certain condition, we find fronts exhibiting an unphysical behavior: they travel faster in the subdiffusive than in the diffusive regime.
\end{abstract}

DOI: 10.1103/PhysRevE.71.057105

\section{INTRODUCTION}

Hyperbolic reaction-diffusion equations have been applied to model memory or delay effects in heat conduction [2] and many other biological applications (see Ref. [1] and references therein). In all these models, memory effects come from assuming a single and small waiting time between successive jumps. From the continuous-time random walk (CTRW) framework the practical procedure consists in expanding in Taylor series the waiting time probability distribution function (PDF) in the Laplace space. Up to first order one gets the classical parabolic reaction-diffusion equation, commonly called Fisher's equation, and up to second order one has the hyperbolic reaction-diffusion equation (see Ref. [1] for details). By considering an anomalous waiting time PDF one finds a fractional reaction-diffusion equation (Ref. [3] and references therein are a complete guide to physics phenomena related to these equations) and up to first order in the Taylor expansion one has a parabolic reactiondiffusion equation. The speed of fronts for these equations has been recently found [4], as well as asymmetric fronts [5] and the Turing conditions for pattern formation [6]. In this work we derive and study a fractional hyperbolic reactiondiffusion (FHRD) equation. We consider an anomalous, that is, a Lévy (i.e., a stable), waiting time PDF often used in the literature [3], and retain terms up to second order in the Taylor expansion. We analyze the speed of fronts in FHRD equations by using the Hamilton-Jacobi equation method [7] and find numerical and approximated solutions to the speed. The Hamilton-Jacobi formalism of classical mechanics has already been widely employed to derive the speed of fronts in hyperbolic reaction-diffusion equations under FisherKolmogorov-Petrovskii-Piskunov (Fisher-KPP) kinetics [8]. We determine here also the limits of the linear speed selection for hyperbolic fronts in terms of the reaction-diffusion dimensionless number $a$ (it is the quotient between the characteristic waiting and reaction times) and the fractional exponent $\gamma$. Within the linear speed selection range we find fronts that travel faster in the subdiffusive regime than in the diffusive one. Our results may be potentially interesting to consider the effect of a time delay in folded polymers, scalefree networks [9], and magnetically confined plasmas [5].
PACS number(s): 05.70.Ln, 05.40.Fb, 02.50.Ey

\section{HYPERBOLIC FRACTIONAL REACTION-DIFFUSION EQUATIONS}

A reaction-dispersal equation has been recently derived according to the CTRW framework [10]. Inverting Eq. (3) in Ref. [10] by a Fourier-Laplace transform and assuming decoupled spatial and temporal memories, one gets

$$
\begin{aligned}
\rho(x, t)= & \int_{0}^{t} d t^{\prime} \varphi\left(t^{\prime}\right) \int_{\mathrm{R}} d x^{\prime} \Phi\left(x^{\prime}\right) \rho\left(x-x^{\prime}, t-t^{\prime}\right) \\
& +\int_{0}^{t} d t^{\prime} \phi\left(t^{\prime}\right) f\left(\rho\left(x, t-t^{\prime}\right)\right),
\end{aligned}
$$

where $\rho(x, t)$ is the concentration of particles at position $x$ and time $t, \varphi(t)$ is the waiting time PDF, and $\Phi(x)$ is the jump length PDF. The survival probability at the current position is $\phi(t)=\int_{t}^{\infty} d t^{\prime} \varphi\left(t^{\prime}\right)$. Finally, $f$ represents the rate of creation or removal of particles which, as usual in population dynamics, depends explicitly on $\rho$ as a nonlinear function. We define $f \equiv r \rho F(\rho)$, where $r$ is the constant growth rate, and consider $f$ of the Fisher-KPP type [11,7], that is, $F(\rho)$ is such that $F(0)=1, F(\rho) \leqslant F^{\prime}(0)$, and $F(\rho)>0$ for $\rho \in(0,1)$. Let us assume a Gaussian jump length $\operatorname{PDF} \Phi(k)=e^{-\sigma^{2} k^{2}}$ and the anomalous version of the single waiting time PDF $\varphi(s)$ $=e^{-(s \tau)^{\gamma}}$ with $0<\gamma \leqslant 1$, which is a Lévy (i.e., stable) distribution. If one chooses other similar anomalous PDFs, these can be written after a Taylor expansion as $\Phi(k)=1$ $-\alpha_{1}(\sigma k)^{2}+\cdots$ and $\varphi(s)^{-1}=1+\beta_{1}(s \tau)^{\gamma}+\frac{1}{2} \beta_{2}(s \tau)^{2 \gamma}+\cdots$ with proper $\alpha_{i}$ and $\beta_{i}$ coefficients [12]. So, our choice does not imply a loss of generality. On setting both PDFs in the Fourier-Laplace transform of Eq. (1) one has

$$
s\left[e^{(s \tau)^{\gamma}}-e^{-\sigma^{2} k^{2}}\right] \rho(k, s)=\left[e^{(s \tau)^{\gamma}}-1\right] f(k, s) .
$$

From now we assume short jump lengths $(\sigma k \ll 1)$ and small waiting times $(s \tau \ll 1)$. These facts allow to us keep the linear terms in the expansion of PDFs, i.e., $\Phi(k) \simeq 1-\sigma^{2} k^{2}$ and $e^{(s \tau)^{\gamma}} \simeq 1+(s \tau)^{\gamma}$. However, dealing with the waiting time PDF, we shall explore the next approximation level and take $e^{(s \tau)^{\gamma}} \simeq 1+(s \tau)^{\gamma}+\frac{1}{2}(s \tau)^{2 \gamma}$. On one hand, by introducing $e^{-\sigma^{2} k^{2}} \simeq 1-\sigma^{2} k^{2}$ and $e^{(s \tau)^{\gamma}} \simeq 1+(s \tau)^{\gamma}$ into Eq. (2), we get (af- 


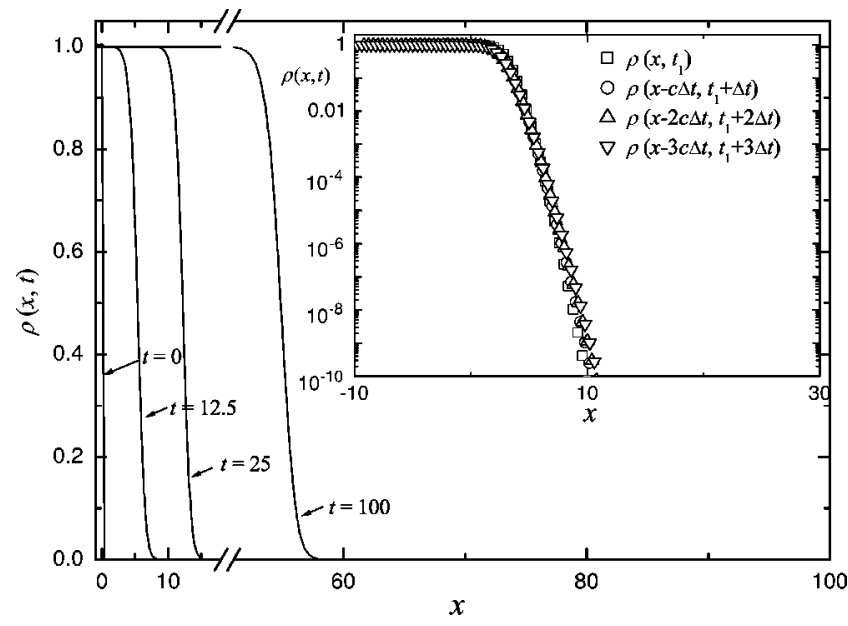

FIG. 1. Front profiles obtained from numerical integrations of Eq. (4). Here we have chosen $\gamma=0.7, a=r \tau=10^{-4}$. Inset: Front profiles collapse by exchanging the variables $(x, t)$ for $(x-c \Delta t, t+\Delta t)$. In this case we have computed $c=0.559$ directly from the numerical calculations [the predicted value from Eq. (12) is 0.551] and $\Delta t$ $=7.5, t_{1}^{*}=7.5$ (squares), $t_{2}^{*}=15$ (circles), $t_{3}^{*}=22.5$ (up triangles), and $t_{4}^{*}=30$ (down triangles). We have omitted the asterisks for notational simplicity.

ter inverting the Fourier-Laplace transform [3] the parabolic fractional reaction-diffusion (PFRD) equation

$$
\partial_{t}^{\gamma} \rho=\frac{\sigma^{2}}{\tau^{\gamma}} \partial_{x x} \rho+\partial_{t}^{\gamma-1} f .
$$

The speed of fronts propagating according to Eq. (3) has been already studied recently [4]. On the other hand, if we introduce $e^{-\sigma^{2} k^{2}} \simeq 1-\sigma^{2} k^{2}$ and $e^{(s \tau)^{\gamma}} \simeq 1+(s \tau)^{\gamma}+\frac{1}{2}(s \tau)^{2 \gamma}$ into Eq. (2) one gets the hyperbolic fractional reaction-diffusion (HFRD) equation

$$
\frac{\tau^{\gamma}}{2} \partial_{t}^{2 \gamma} \rho+\partial_{t}^{\gamma} \rho=D \partial_{x x} \rho+\frac{\tau^{\gamma}}{2} \partial_{t}^{2 \gamma-1} f+\partial_{t}^{\gamma-1} f
$$

where we have defined the diffusion coefficient $D \equiv \sigma^{2} / \tau^{\gamma}$. In this work we focus on fronts arising from Eq. (4). Let us stress that in the absence of reaction $(f=0)$ the transport regime in this case is always subdiffusive in the large time limit (see Ref. [13] for details). In order to check the existence of fronts as solutions of Eq. (4) we have integrated it numerically by using a finite difference scheme based in the equivalence of the Riemann-Liouville and GrünwaldLetnikov derivatives [14], in the same way as appear in Ref. [15]. We have also got the following stability condition for the algorithm to converge:

$$
\frac{a^{2 \gamma-1}\left(\Delta x^{*}\right)^{2}+4\left(\Delta t^{*}\right)^{2 \gamma}}{2 a^{\gamma-1}\left(\Delta x^{*}\right)^{2}\left(\Delta t^{*}\right)^{\gamma}}<1,
$$

where $\left(\Delta x^{*}\right)^{2}$ and $\left(\Delta t^{*}\right)^{2}$ are the grid spacing along the $x^{*}$ and $t^{*}$ axes, respectively. Figure 1 shows numerical solutions of Eq. (4) for several times from a steep initial condition. We also plot (inset in Fig. 1) the collapse of these profiles when an appropriate change of variables is made.

\section{SPEED OF HFRD FRONTS}

The Hamilton-Jacobi equation corresponding to the problem (1) under an initial condition with compact support is (see Ref. [10])

$$
\frac{1}{\hat{\varphi}(H)}=\hat{\Phi}(p)+\frac{r}{H}\left(\frac{1}{\hat{\varphi}(H)}-1\right)
$$

where $\hat{\varphi}(H)$ and $\hat{\Phi}(p)$ are nothing but the Laplace transform and the bilateral transform of the waiting time and the jump length distributions, respectively. The speed of the front is determined by the system of algebraic equations

$$
v=\frac{H}{p}, \quad \frac{d H}{d p}=\frac{H}{p},
$$

where $H=H(p)$ is obtained from Eq. (5). On putting $\hat{\varphi}(H)$ $=e^{-(H \tau)^{\gamma}}$ and $\Phi(p)=e^{\sigma^{2} p^{2}}$ into Eq. (5) it reads as

$$
e^{(H \tau)^{\gamma}}=e^{\sigma^{2} p^{2}}+\frac{a}{H \tau}\left[e^{(H \tau)^{\gamma}}-1\right]
$$

where $a=r \tau$ is the dimensionless reaction-diffusion number. This dimensionless parameter expresses the quotient between the characteristic waiting time and the characteristic reaction time. Up to first order in $H \tau$ and in $\sigma^{2} p^{2}$ one obtains the Hamilton-Jacobi for the PFRD equation and the speed has been already found in [16]. Up to second order in $H \tau$ one has $e^{(H \tau)^{\gamma}} \simeq 1+(H \tau)^{\gamma}+\frac{1}{2}(H \tau)^{2 \gamma}$ and up to first order in $\sigma^{2} p^{2}$, Eq. (7) reads

$$
\sigma^{2} p^{2}=(H \tau)^{\gamma}\left[1+\frac{1}{2}(H \tau)^{\gamma}\right]\left(1-\frac{a}{H \tau}\right)
$$

which requires $H \tau>a$ in order to have physical meaning. By virtue of the first equation in (6) and (8) the speed of the front is given by

$$
v_{\gamma}=\frac{\sigma}{\tau} \frac{y^{(3-\gamma) / 2}}{\sqrt{y-a} \sqrt{1+y^{\gamma / 2}}}
$$

where $y \equiv H \tau$ is, from the second equation in (6), a solution of the transcendent equation

$$
(1-\gamma) y^{\gamma+1}-\frac{a}{2}(3-2 \gamma) y^{\gamma}+(2-\gamma) y-a(3-\gamma)=0
$$

As Eq. (10) has no analytical solution we propose to find an approximated solution by assuming a perturbative expansion around $y=a$, that is, we insert $y=a+\epsilon$ in (10) and find up to first order in $\epsilon$

$$
\epsilon \simeq \frac{2 a\left(1+a^{\gamma / 2}\right)}{(2-3 \gamma) a^{\gamma}+2(2-\gamma)}
$$

Therefore, the approximated solution for the speed is from Eqs. (9) and (11) 
TABLE I. Comparison of the speed from Eq. (12) $\left(c_{\text {theor }}\right)$ and from numerical integrations of Eq. (4) ( $\left.c_{\text {num }}\right)$ for $a=10^{-4}$.

\begin{tabular}{ccc}
\hline \hline$\gamma$ & $c_{\text {theor }}$ & $c_{\text {num }}$ \\
\hline 0.3 & 0.095 & 0.117 \\
0.5 & 0.231 & 0.266 \\
0.7 & 0.552 & 0.559 \\
0.99 & 1.917 & 1.880 \\
\hline \hline
\end{tabular}

$$
\begin{aligned}
v_{\gamma} \simeq & \frac{\sigma}{\tau}\left[\frac{(2-3 \gamma) a^{\gamma}+2(2-\gamma)}{2 a\left(1+\frac{a^{\gamma}}{2}\right)}\right]^{1 / 2}[G(a, \gamma) a]^{(3-\gamma) / 2} \\
& \times\left\{1+\frac{[G(a, \gamma) a]^{\gamma}}{2}\right\}^{-1 / 2} \\
& \text { with } G(a, \gamma)=\frac{3 a^{\gamma}(1-\gamma)+2(3-\gamma)}{(2-3 \gamma) a^{\gamma}+2(2-\gamma)} .
\end{aligned}
$$

As $H \tau>a$, one must demand that $\epsilon>0$. Thus, the conditions for the existence of a linear speed selection for fronts in HFRD equations is from Eq. (11)

$$
a<a_{c}(\gamma)=\left(\frac{4-2 \gamma}{3 \gamma-2}\right)^{1 / \gamma} \quad \text { if } \gamma>\frac{2}{3} .
$$

For $\gamma \leqslant 2 / 3$ no condition is required. Let us to stress that for the classical case $\gamma=1$ one has from Eq. (10) $y=4 a /(2-a)$ and from Eqs. (9) and (13)

$$
v_{1}=4 \frac{\sigma}{\tau} \frac{\sqrt{a}}{2+a} \quad \text { if } a<2 .
$$

This result is already known for hyperbolic reactiondiffusion equations (1). In Table I we compare the results of the speed from Eq. (12) and the speed we have calculated from the numerical integration of Eq. (4). We find a good agreement for all the values of $\gamma$. It is interesting to find the set of values for $a$ and $\gamma$ for which the linear speed selection holds and the correlation between the transport regime and the speed of the front. This is illustrated in Fig. 2. For values of $a$ higher than $a^{*}(\gamma)$, the linear speed of the fronts is also selected but fronts can travel faster for $\gamma<1$ than for $\gamma=1$. This is inconsistent with the fact that for $\gamma<1$ the transport is subdiffusive, i.e., slower than in the transport regime for $\gamma=1$ (normal diffusion). Fronts with $a>a^{*}(\gamma)$ lack physical meaning.

\section{COMPARISON WITH OTHER APPROACHES}

In the context of front speed, we discuss the improvement achieved by using HFRD over PFRD equations in this section. For this purpose, we need to deal with the complete jump length PDF, that is, we need to consider the infinite order in the Taylor expansion of $\hat{\Phi}(p)$. Otherwise, a front cannot exist if one considers the complete tail for the waiting time PDF. This was shown in Ref. [16] where we studied the linear speed selection for fronts (pulled fronts) in the CTRW

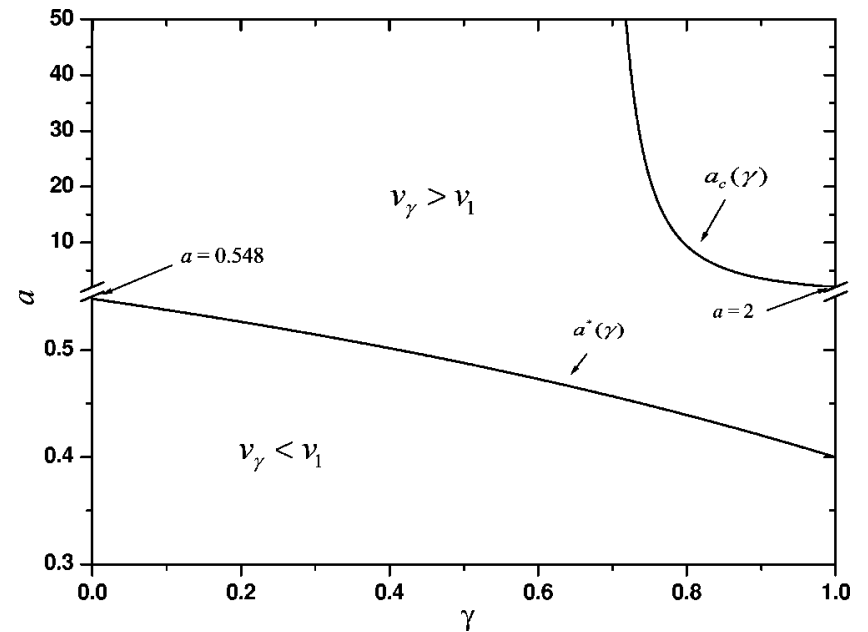

FIG. 2. Plot for the critical reaction-diffusion dimensionless number versus the fractional index $\gamma$ given in Eq. (13). When the values of $a$ and $\gamma$ lie over the curve $a_{c}(\gamma)$ given in Eq. (13) no linear speed is selected. The curve $a^{*}(\gamma)$ represents the separatrix of the domain where $v_{\gamma}>v_{1}$ and $v_{\gamma}<v_{1}$.

framework. If $N$ is of the order of the truncation of the Taylor series in $s \tau$ of the inverse of the Laplace transform of the waiting time $\operatorname{PDF}\left[\varphi(s)^{-1}\right]$ and $M$ is of the order of the truncation of the Taylor series in $\sigma p$ of the bilateral transform of the jump length PDF $[\Phi(p)]$, it is shown in [16] that only for $N \leqslant M$ is the minimum linear speed selected. From Eqs. (6) and (7) one obtains

$$
v_{e x}=\frac{\sigma}{\tau_{y>a}} \min \frac{y}{\ln ^{1 / 2}\left[e^{y^{\gamma}}(1-a / y)+a / y\right]} .
$$

The solution of $v_{e x}$ has to be numerically obtained. Now we can make two approximations to the previous exact result. Up to first order we take $e^{(H \tau)^{\gamma}} \simeq 1+(H \tau)^{\gamma}$ which corresponds to the parabolic approach. Then, from Eqs. (6) and (7)

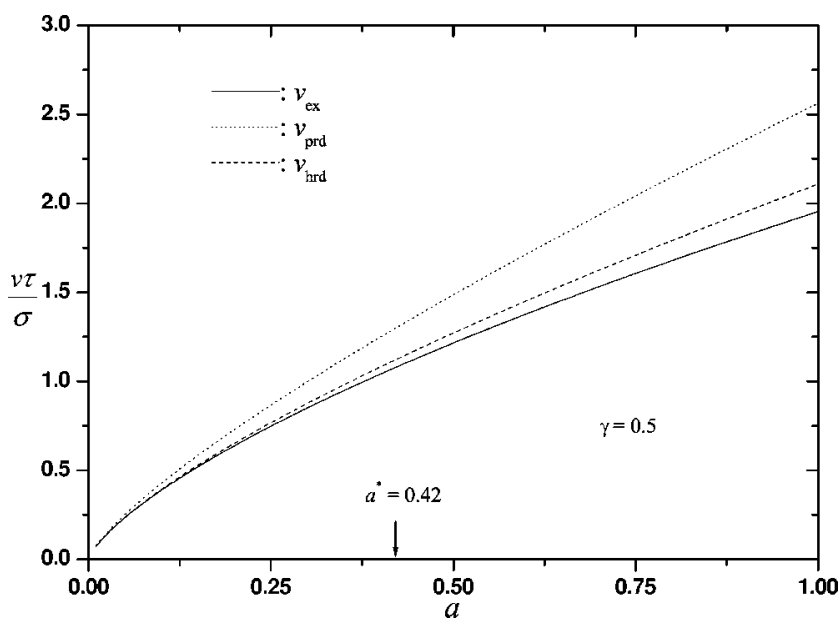

FIG. 3. The dimensionless speed $v \tau / \sigma$ is plotted versus $a$. A monotonically increasing behavior of the speed and a considerable improvement of the hyperbolic approach in comparison to the parabolic approach is observed. 


$$
v_{p r d}=\frac{\sigma}{\tau_{y>a}} \operatorname{\operatorname {ln}^{1/2}[1+y^{\gamma }-ay^{\gamma -1}]} .
$$

Up to second order, $e^{(H \tau)^{\gamma}} \simeq 1+(H \tau)^{\gamma}+\frac{1}{2}(H \tau)^{2 \gamma}$ we get the hyperbolic approach and the speed of the front is from Eqs. (6) and (7)

$$
v_{h r d}=\frac{\sigma}{\tau \min _{y>a}} \frac{y}{\ln ^{1 / 2}\left[1+y^{\gamma}+\frac{1}{2} y^{2 \gamma}-a\left(y^{\gamma-1}+\frac{1}{2} y^{2 \gamma-1}\right)\right]} .
$$

In Fig. 3 we plot the numerical computations for the speed of the fronts coming from these three different cases (15), (16), and (17). It is observed that the parabolic approach considerably deviates from the "exact case" while the hyperbolic approach is a good approach for small values of $a$ (as it must be in order to get fronts with physical meaning), improves substantially the parabolic approach, and allows us to think that the hyperbolic approach retains most of the memory contained in the complete form of the waiting time PDF and has the advantage that it is still analytically treatable.

\section{CONCLUSIONS}

We have proved numerically the existence of a traveling wave front solution and have studied the speed of fronts for FHRD equations. We have found numerical and approximated solutions for the speed exhibiting a good agreement. Conditions for the existence of a linear front speed selection in terms of the reaction-diffusion dimensionless number $a$ and the fractional index $\gamma$ have been found. We have also mathematically shown, within the linear speed selection, the emergence of fronts driven by subdiffusion transport, that can travel, if $a>a^{*}(\gamma)$, faster than fronts driven by classical diffusion. However, this mathematical result has no physical meaning. Finally, we have compared the parabolic approach with the result obtained from the complete waiting time PDF (called the exact case) and a notable deviation has been observed. However, when the hyperbolic approach is considered, the agreement with the exact case is substantially improved within the range of values of $a$ where the fronts have physical meaning.

\section{ACKNOWLEDGMENTS}

This work has been supported by the MCYT under Grant No. BFM 2003-06033.
[1] J. Fort and V. Méndez, Rep. Prog. Phys. 65, 895 (2002).

[2] D. Jou, J. Casas-Vázquez, and G. Lebon, Extended Irreversible Thermodynamics (Springer, Berlin, 2001).

[3] R. Metzler and J. Klafter, Phys. Rep. 339, 1 (2000).

[4] S. Fedotov and V. Méndez, Phys. Rev. E 66, 030102(R) (2002).

[5] D. del-Castillo-Negrete, B. A. Carreras, and V. E. Lynch, Phys. Rev. Lett. 91, 018302 (2003).

[6] B. I. Henry and S. L. Wearne, Physica A 276, 448 (2000).

[7] M. Freidlin, Functional Integration and Partial Differential Equations (Princeton University Press. Princeton, NJ, 1985).

[8] S. Fedotov, Phys. Rev. E 59, 5040 (1999); 60, 4958 (1999); J. Phys. A 33, 7033 (2000).

[9] D. Brockmann and T. Geisel, Phys. Rev. Lett. 90, 170601 (2003).
[10] V. Méndez, D. Campos, and S. Fedotov, Phys. Rev. E 70, 036121 (2004).

[11] J. D. Murray, Mathematical Biology (Springer-Verlag, Berlin, 1993).

[12] An alternative selection could be, for example, the Laplace $\operatorname{PDF} \Phi(x)=(2 \sigma)^{-1} e^{-|x| / \sigma}$ with Fourier transform $\Phi(k)=(1$ $\left.+\sigma^{2} k^{2}\right)^{-1}$ and anomalous waiting time PDF $\varphi(s)=[1$ $\left.+(s \tau)^{\gamma}\right]^{-1}$. In this case, $\alpha_{i}=(-1)^{i}, \beta_{1}=1$, and $\beta_{i>1}=0$.

[13] A. Compte and R. Metzler, J. Phys. A 30, 7277 (1997).

[14] S. G. Samko, A. A. Kilvas, and O. I. Marichev, Fractional Integrals and Derivatives: Theory and Applications (Gordon and Breach Science Publishers, New York, 1993).

[15] M. Ciesielski and J. Leszczynski, e-print math-ph/0309007.

[16] V. Méndez, D. Campos, and S. Fedotov, Phys. Rev. E 70, 036121 (2004). 